\title{
Detection Methods of Interharmonics Parameters Based on DFT Filter Banks
}

\author{
Quanyi Jin \\ School of Mechanical Engineering, \\ Shanghai University of Engineering \\ Science, Shanghai ,China
}

\begin{abstract}
There are a large number of interharmonics in electric power system. But if FFT algorithm is directly used for interharmonic analysis, it exists spectrum leakage and hurdle effect. This paper presents using multi-sample rate digital signal processing technology to realize narrowb and low-pass filter, on this basis, using the DFT filter banks to detect interharmonic frequency and amplitude. The simulation results show, the new algorithm has high detection precision.
\end{abstract}

\section{Keywords}

DFT filter bank ; interharmonics ; multi-sample rate

\section{INTRODUCTION}

Non-linear load of semiconductor devices are widely used in electric power distribution system. Because their nonlinear load characteristics, the electric power system exists many interharmonics. Current research shows that interharmonics voltage must be restrained to very low enough level. The study of harmonics is very important topic for electric power quality. Research topics include harmonic source analysis and efforts, measurement technology and elimination strategy.

But when we use FFT to analysis interharmonics, it exists spectrum leakage and hurdle effect.

\section{DFT FILTER BANKS}

When a filter bank is used to calculating the discrete Fourier transform of signal, it is the DFT filter bank. In theory, A full band signal by DFT filter bank is divided into a series of narrow band signals.

In the M-channel filter bank Analyzer, Input signal $x(n)$ is divided into channel of $\mathrm{M}$ signal. As shown in Figure 1, $X_{k}(m)$ represents the multiple signals, which $\mathrm{k}=0,1 \ldots \mathrm{M}-1$.

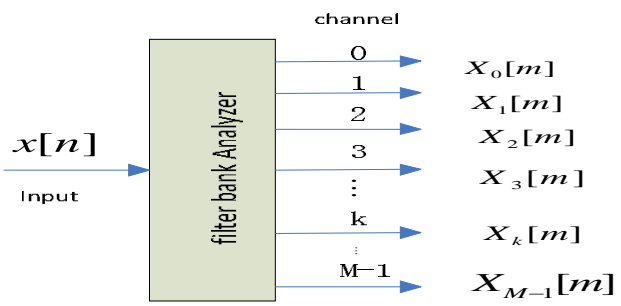

Figure 1. The basic structure of M-channel filter bank Analyzer
In the filter bank synthesizer, The input signal is $\hat{X}_{k}(m)$, which $\mathrm{k}=0,1 \ldots \mathrm{M}-1 . \hat{x}(n)$ stands for the output signal of the synthesizer. As shown in Figure 2.

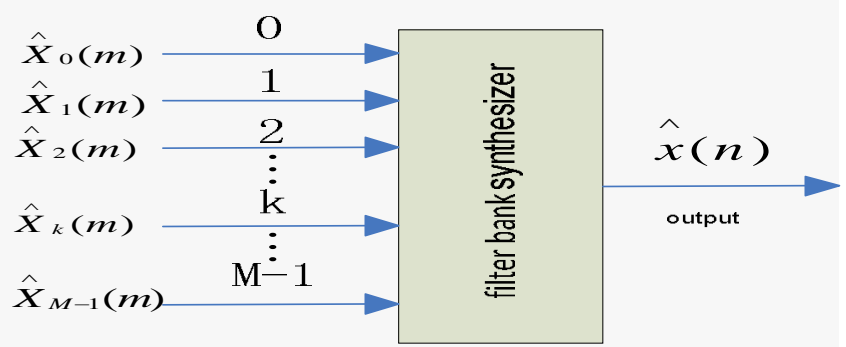

Figure 2. The basic structure of M-channel filter bank synthesizer

For a DFT filter bank, It's the Kth channel as shown in Figure 3.

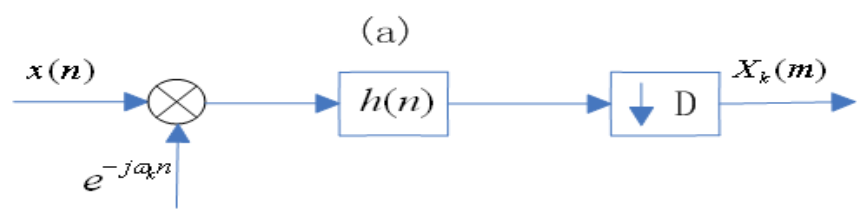

(b)

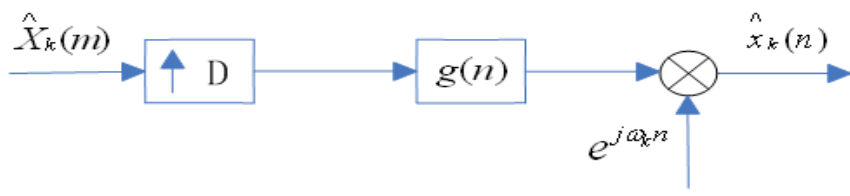

Figure 3 (a) Single channel of DFT filter bank analyzer (b) Single channel of DFT filter bank synthesizer

The channel signal of DFT filter bank analyzer can be expressed as

$X_{k}(m)=\sum_{n=-\infty}^{\infty} h(m D-n) x(n) e^{-j \omega_{k} n} \quad k=0,1, \cdots M-1$

The channel signal of DFT filter bank synthesizer can be expressed as

$$
\hat{x}_{k}(n)=e^{j \omega_{k} n} \sum_{m=-\infty}^{\infty} \hat{X}_{k}(m) g(n-m D) \quad k=0,1, \cdots M-1
$$




\section{Detection Methods of Interharmonics Parameters Based on DFT Filter Banks}

The output of DFT filter bank synthesizer is a sum of all channel

$$
\begin{aligned}
& \text { signals } \hat{x}_{k}(n) \text {. } \\
& \hat{x}(n)=\sum_{m=-\infty}^{\infty} g(n-m D) \frac{1}{M} \sum_{k=1}^{M-1} \hat{X}_{k}(m) e^{j \omega_{k} n}
\end{aligned}
$$

\section{DETECT INTERHARMONICS}

DFT filter bank has M Channels. It's composed of M low-pass filters (LPF) . As shown in Figure 4, by DFT filter banks, discrete time signal $x(n)$ is divided into M narrow-band signals $x_{k}[n]$ of equivalent rate ( Bandwidth is $2 \mathrm{~Hz}$ ), which $\mathrm{k}=0,1 \ldots \mathrm{M}-1$. The center frequency is $\omega_{k}=2 \pi k / M$, When the signal $x(n)$ through LPF, the low frequency part in the spectrum is extracted . When any of the harmonic waves in the signal $x(n)$ are needed to extract (for example, the center frequency is $\omega=\omega_{k}$ part), Signals $x(n)$ can be multiplied by $e^{-j n \omega_{k}}$, So the band is modulated to the baseband, then you can extract the harmonic information by LPF.

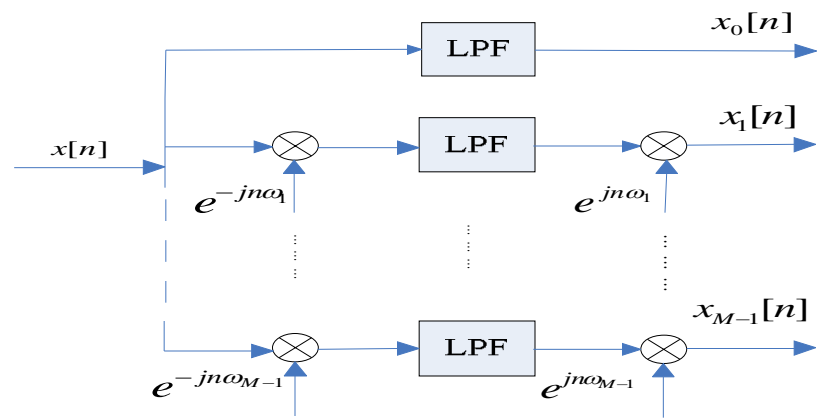

Figure 4. Basic block diagram of DFT filter bank

The basic process of Interharmonics detection is as follows. First, when the analog signal goes through the anti-aliasing filter, the high frequency part of the signal is filtered out $(>5 \mathrm{kHz})$. Then the analog signal goes through the A/D converter, we can get a set of discrete time-domain signal $x(n)$. This group signal gets interharmonic information by DFT filter banks. The information contains components of time domain and frequency domain.

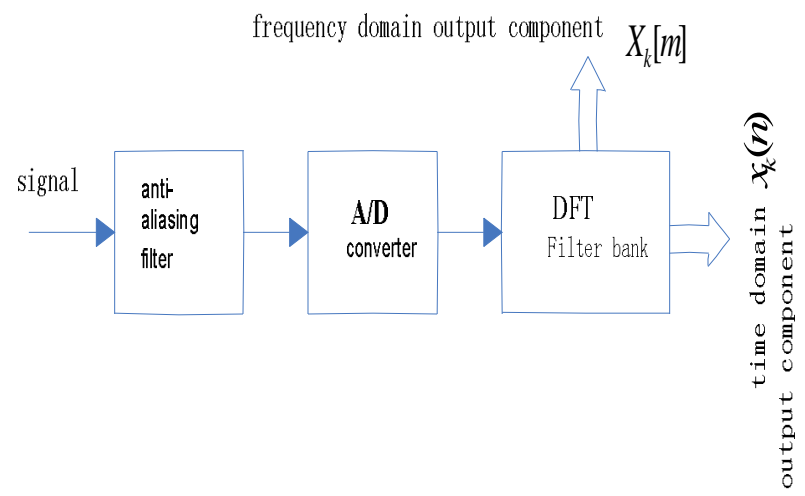

Figure 5. The basic process of interharmonics detection

\section{SIMULATION ANALYSIS}

Assuming that a voltage signal in the electrical system as shown in Figure 6. It uses DFP filter banks to filter, the sampling rate is $\mathrm{F}=10 \mathrm{kHz}$. There is interharmonic domain component in four channels.

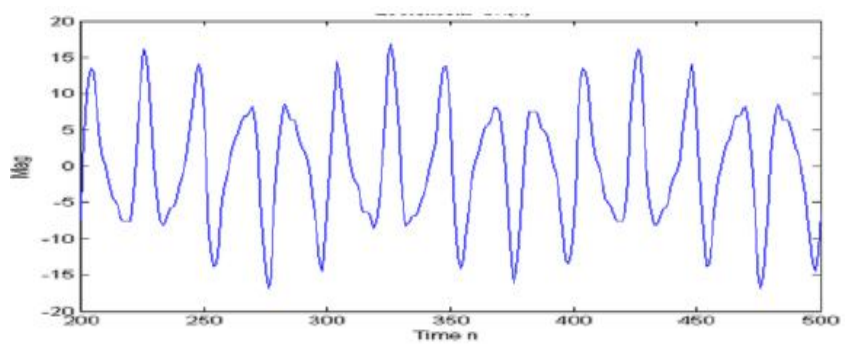

Figure 6. One voltage signal of power system

They are the 29th channel, the 45th channel, the 68th channel and the 118th channel. The passband edge frequency of narrowband lowpass filter is $2 \mathrm{~Hz}$. We can detect 4 interharmonics in the voltage signal. Their parameters are as follows:

The 29th channel: interharmonic frequency is $57 \mathrm{~Hz}$, amplitude is 10.The 45th channel: interharmonic frequency is $89 \mathrm{~Hz}$, amplitude is 5.The 68th channel: interharmonic frequency is $135 \mathrm{~Hz}$, amplitude is 1.5.The 118th channel: interharmonic frequency is $235 \mathrm{~Hz}$, amplitude is 0.5 .

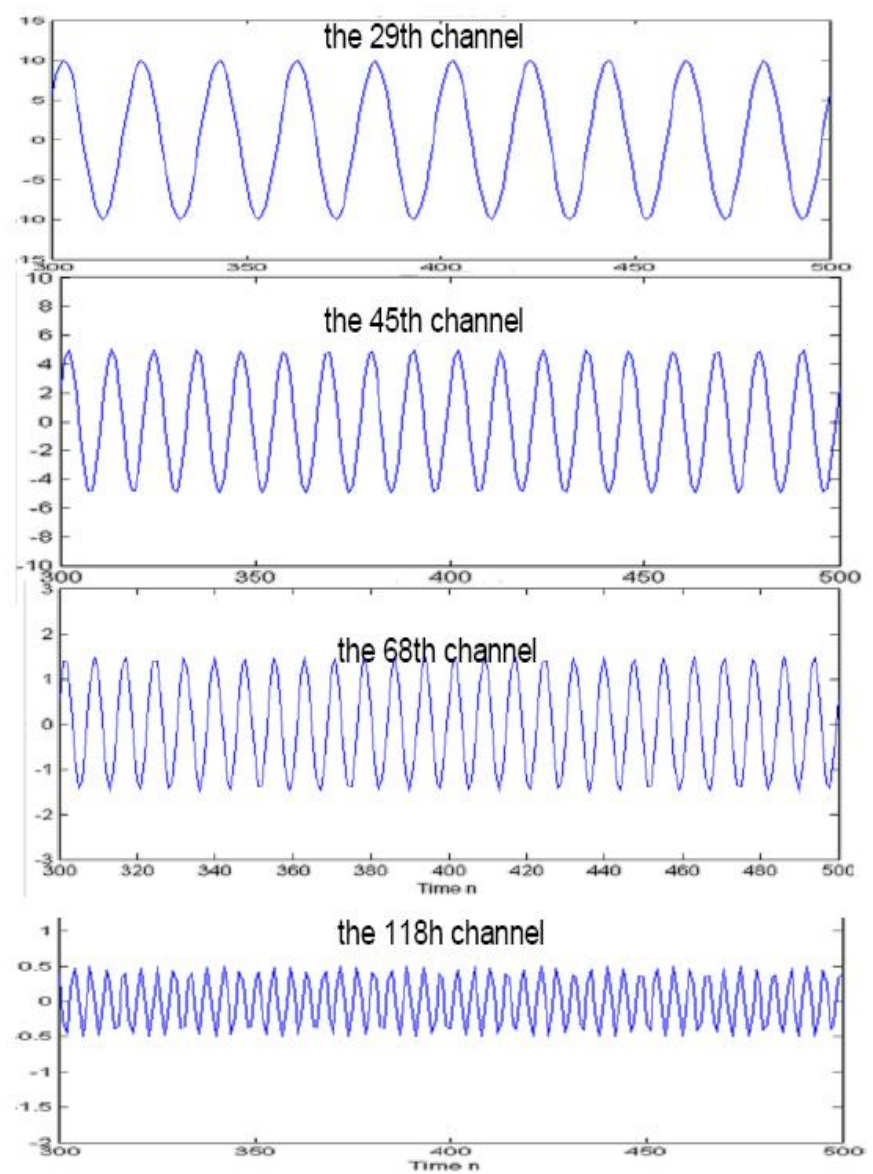

Figure 7. Each channel signal waveform 


\section{CONCLUSIONS}

As can be seen from the results of the analysis, it is very easy to detect the parameters of harmonics and interharmonics in electric power system by using DFT filter banks. The similating result shows that the new method reaches a higher accuracy.

\section{REFERENCES}

[1] R. Yacamini, Power system harmonics. iv. interharmonics," Power Engineering Journal [see also Power Engineer], vol. 10, no. 4, pp. 185-193,Aug. 1996.

[2] F. de Rosa, R. Langella, A. Sollazzo, and A. Testa, IOn the interharmonic components generated by adjustable speed drives," Power Delivery, IEEE Transactions on, vol. 20, no. 4, pp. 2535-2543, Oct. 2005.

[3] General guide on harmonics and interharmonics measurements," IEC61000-4-7, 2000.
[4] D. Basic, V. S, Ramsden and P. K. Muttik, "Hybrid filter control system with adaptive filters for selective elimination of harmonics and interbarmonics", IEE Proc. - Electr. Power Appl., May 2000, Vol. 147 No.3, 295 303

[5] J. D. Johnston, “Audio coding with filter banks,"in Subband and Wavelet Transform, Akansu. A. and Smith, M., Eds., Kluwer Academic Press, 2006

[6] Mohammed Bashir Rifai, Thomas H. Ortmeyer, Senior Member, IEEE, and William J. McQuillan, "Evaluation of CurrentInterharmonics from AC Drives", IEEE TRACTIONS ON POWER DELIVERY, July 2000 , VOL.15, N0.3, $1094-1098$ 